\title{
Influência das características geológicas do local de desova na duração da incubação e no sucesso da eclosão dos ovos de Caretta caretta na praia da Guanabara, Anchieta, Espírito Santo
}

\author{
Paulo D. Ferreira Júnior ${ }^{1}$, Maria F. Rosa², Mariângela De Lorenzo ${ }^{3}$, \\ Matheus F. Monteiro ${ }^{4} \&$ Romildo A. Júnior ${ }^{5}$
}

\begin{abstract}
1. Programa de Mestrado em Ecologia de Ecossistemas, Centro Universitário Vila Velha, Rua Comissário Jose Dantas de Melo, 21, Boa Vista, 29102-770 Vila Velha, ES. (pdfj@hotmail.com)

2. Projeto TAMAR/IBAMA, Base de Anchieta, Avenida Beira Mar, 6, 29230-000 Anchieta, ES. (maria.tamar@terra.com.br)

3. Autônoma. Rua Coronel Monjardin 165/1102, Centro, 29.015-500 Vitória, ES. (mariangeladelorenzo@ig.com.br)

4. Curso de Engenharia Ambiental, Universidade Federal de Ouro Preto, Rua do Paraná, 54, Centro, 35400-000 Ouro Preto, MG (matheusfunchal@gmail.com)

5. Centro Universitário Vila Velha, Rua Comissário Jose Dantas de Melo, 21, Boa Vista, 29102-770 Vila Velha, ES. (romildoazevedo@ hotmail.com)
\end{abstract}

\begin{abstract}
Influence of geological characteristics on the incubation duration and hatchling success of Caretta caretta on the Guanabara Beach, Anchieta, Espírito Santo. This study analyzed the possible influences of nest site on hatch success and incubation duration of Caretta caretta (Linnaeus, 1758) in the south of Espírito Santo, Brazil. Geomorphologically, the nest site on Guanabara beach, in Anchieta (ES), may be divided into open beach and berm. During four nesting seasons, data on sediment size, nest height from sea level, geomorphological compartmentation of the beach, date of laying, number of living hatchlings and clutch size were collected. Results showed variations of the main sediment parameters between the years, indicating that nesting seasons should be analyzed individually. Hatch success was not affected by nest site characteristics. Incubation duration did not vary between beach and berm, despite the significant differences in sediment size, nest depth and height from sea level. Incubation duration correlated positively with sediment size, with nests in coarser sediments hatching earlier than the ones in finer sediments. Since nest characteristics influence incubation duration, it is recommended that nest transfers, when inevitable, respect the differences between nest sites, especially, concerning nest depth.
\end{abstract}

KEYWORDS. Loggerhead sea turtle, nest, beach, berm.

RESUMO. O objetivo deste estudo foi analisar possíveis influências do local da desova no sucesso da eclosão e na duração da incubação de Caretta caretta (Linnaeus, 1758) no sul do estado do Espírito Santo. Geomorfologicamente a área da desova na praia da Guanabara, em Anchieta (ES), pode ser dividida em praia aberta e berma. Durante quatro estações reprodutivas foram coletados dados sobre o tamanho dos sedimentos, a altura dos ninhos em relação ao nível do mar, a compartimentação geomorfológica da praia, a data da desova, o número de filhotes vivos e o tamanho da ninhada. Os resultados mostraram variações dos principais parâmetros sedimentológicos entre os anos, indicando que as temporadas reprodutivas devem ser analisadas individualmente. O sucesso da eclosão não foi afetado pelas características geológicas do local da desova. A duração da incubação não variou entre a praia e a berma, apesar das diferenças significativas no tamanho do sedimento, na profundidade dos ninhos e na altura em relação ao nível do mar. A duração da incubação se correlacionou positivamente ao tamanho dos sedimentos, com ninhos em sedimentos mais grossos eclodindo mais cedo que os ninhos localizados em sedimentos mais finos. Como as características dos ninhos influenciam a duração da incubação, recomenda-se que a transferência de ninhos, quando inevitável, respeite as diferenças entre os locais de desova, principalmente, quanto à profundidade da câmara de ovos.

PALAVRAS-CHAVE. Tartaruga, sedimento, reprodução, praia, berma.

Todas as tartarugas marinhas apresentam determinação sexual dependente da temperatura da incubação (WibBELs, 2003). Isso significa que o sexo dos filhotes será influenciado pela temperatura da incubação que, por sua vez, depende de diversos fatores ambientais. Esta premissa tem importantes conseqüências nos estudos sobre a ecologia reprodutiva das tartarugas marinhas e nos programas de conservação que envolvem o manejo e a transferência de ninhos (Milton et al., 1997; NARo-Maciel et al.,1999; Baptistotte et al., 1999). Na temperatura pivotal (Mrosovsky \& YNTEMA, 1980) a proporção é de $50 \%$ para cada um dos sexos; à medida que as temperaturas aumentam, uma maior proporção de fêmeas é gerada; inversamente, a diminuição da temperatura ocasiona uma maior proporção de machos (Mrosovsky et al., 1984; BAPTISTOTTE et al., 2003). Existem limites críticos nos dois extremos do espectro de temperaturas, a partir dos quais o desenvolvimento dos embriões é inviabilizado (MILLER, 1997). Um dos grandes desafios dos estudos de campo está em determinar e quantificar a influência que cada fator ambiental exerce no desenvolvimento embrionário, pois eles interagem de forma complexa e complementar. Os fatores controladores da temperatura a que os ovos serão submetidos podem ser associados a pelo menos três grupos relacionados ao clima, às propriedades fisiográficas do local da desova e à cobertura vegetal. Por exemplo, períodos de grande pluviosidade podem reduzir o sucesso da eclosão devido ao alagamento dos ninhos ou à redução da temperatura da incubação abaixo daquela suportada pelos embriões (Fowler, 1979; Mortimer, 1990; Bobyn \& BroOKs, 1994). A característica mais marcante é o aumento na proporção de fêmeas ao longo de uma mesma estação reprodutiva (Mrosovsky et al., 1984; BAртіSTOTTE et al., 2003). Caretta 
caretta (Linnaeus, 1758) inicia a desova no princípio da primavera e esta se prolonga por todo o verão. Com a proximidade do verão ocorre o aumento da temperatura do ar e do número de horas de sol levando ao nascimento de filhotes com uma razão sexual cada vez mais favorável às fêmeas (Mrosovsky \& Provancha, 1989). A distribuição longitudinal também provoca variações na razão sexual devido a variações no clima. No litoral brasileiro, por exemplo, os filhotes nascidos de desovas de $C$. caretta no estado do Espírito Santo (Praia de Regência: $19^{\circ} 40^{\prime}$ S e 3950'W), apresentam uma razão sexual com uma proporção de machos maior que entre os nascidos no estado da Bahia, ao norte (Praia do Forte: $12^{\circ} 34^{\prime} \mathrm{S}$ e $38^{\circ} \mathrm{W}$ ) (MARCOVAldi et al., 1997; MrosovsKy et al., 1999; BAPTISTOTTE et al., 2003).

A cobertura vegetal pode influenciar a temperatura dos ninhos diretamente através do sombreamento ou, indiretamente, alterando a profundidade da câmara de ovos (Bustard \& GReEnham, 1968). Ninhos de Chelonia mydas (Linnaeus, 1758) (Spotila et al., 1987) e C. caretta (Foley et al., 2000) situados em áreas vegetadas resultaram em um maior número de machos que aqueles localizados nas zonas ensolaradas. A vegetação rasteira, Ipomea pescaprea (Convolvulaceae) e Blutaparon sp. (Amaranthaceae), facilita o processo de abertura dos ninhos de $C$. caretta e os ninhos situados na zona vegetada são mais rasos que aqueles localizados na face de praia, pois as raízes conferem uma maior coesão aos sedimentos e evitam o colapso da câmara de ovos (FERREIRA Júnior et al., 2003).

As propriedades dos sedimentos, como o tamanho, o grau de seleção, a composição mineralógica, o albedo, a salinidade e o pH, são apontadas como elementos que influenciam a escolha das áreas de desova e que afetam a duração da incubação das tartarugas marinhas (GARMESTANi et al., 2000; HAYs et al., 2001). Chelonia mydas que desova em sedimentos mais grossos apresenta um menor sucesso de eclosão na Ilha Ascensão (MorTIMER, 1990). Eretmochelys imbricata (Linnaeus, 1758) (Horrocks \& ScotT, 1991) e C. caretta (HAYs \& SPEAKMAN, 1993) elegem os pontos da praia onde o sucesso da eclosão é maior e onde a praia é mais plana e de menor energia (WoOD \& BJORDNAL, 2000). Na praia de Sekania, Grécia, $C$. caretta desova, preferencialmente nas partes da praia onde a areia é bem selecionada (KARAVAS et al., 2005). MiLTon et al. (1997) mostraram que ninhos de $C$. caretta situados em sedimentos carbonáticos podem ser até $3,4{ }^{\circ} \mathrm{C}$ mais frios que os ninhos situados em sedimentos quartzosos. O albedo (Hays et al., 2001) e a coloração dos sedimentos (NARO-MACIEL et al., 1999) também afetam a temperatura de incubação de $C$. caretta.

O consenso sobre a influência destes diversos fatores está longe de ser alcançado nos trabalhos que abordam o tema. Além das diferenças das praias e dos locais escolhidos para a desova por cada espécie, as diferentes metodologias empregadas em cada trabalho dificultam as correlações e as generalizações. Entender como os elementos relacionados ao sucesso da eclosão e à duração da incubação interagem e quantificar a participação de cada um deles ainda demandará um grande volume de trabalho.
Este trabalho relata a influência das características fisiográficas (tamanho dos sedimentos, altura dos ninhos em relação ao nível do mar e a compartimentação geomorfológica da área de desova) da praia da Guanabara, Anchieta, sul do estado do Espírito Santo, no sucesso da eclosão e na duração da incubação de $C$. caretta durante quatro anos.

\section{MATERIAL E MÉTODOS}

A praia da Guanabara localizada em Anchieta, no sul do estado do Espírito Santo (2050's e 40³7’ W), sudeste do Brasil, conta com uma Base de proteção e monitoramento do Projeto TAMAR-IBAMA desde 2003. De 2000 a 2003 o monitoramento foi realizado pelo Projeto TAVIVAMAR, uma parceria entre a Prefeitura Municipal de Anchieta e IBAMA. O clima é tropical úmido com verões úmidos e invernos secos. A temperatura média anual é de $24^{\circ} \mathrm{C}$ e a pluviosidade gira em torno de 1.200 $\mathrm{mm} / \mathrm{ano}$.

Em 2000 a praia de Anchieta, que possui cerca de $1.500 \mathrm{~m}$, foi mapeada na escala de 1:10.000 com o emprego de bússola e trena. O levantamento topográfico nesta escala permitiu que além da distribuição dos ninhos em cada estação reprodutiva fossem levantadas as principais feições geomorfológicas, afloramentos rochosos e intervenções antrópicas. A praia aberta é desprovida de vegetação e a berma é marcada pelo início de uma vegetação rasteira e espaçada. Um pequeno desnível topográfico, formado pelo acúmulo de sedimentos transportados pelo vento e depositados graças à ancoragem fornecida pelas plantas, destaca a berma, que se constitui de uma faixa alongada paralela à linha de costa. A altura dos ninhos em relação ao nível do mar foi tomada com um teodolito de $5 \mathrm{~mm}$ de precisão. A altura dos ninhos foi medida na maré baixa e os valores encontrados foram corrigidos com a tábua de marés do Porto de Ubu, situado a $5 \mathrm{~km}$ de distância da praia. Este procedimento permitiu que as medidas de todas as temporadas reprodutivas fossem comparadas. A delimitação entre a praia aberta e a berma foi dada pela presença da vegetação e pelas características sedimentológicas.

O tamanho dos sedimentos de cada ninho foi determinado para amostras coletadas a $10 \mathrm{~cm} \mathrm{de}$ profundidade durante a abertura dos ninhos para a verificação do sucesso da eclosão. Uma amostra com cerca de $200 \mathrm{~g}$ foi peneirada nas frações seixo (> $4 \mathrm{~mm})$, grânulo $(2 \mathrm{~mm})$, areia muito grossa (1 mm), areia grossa $(500 \mu \mathrm{m})$ areia média $(250 \mu \mathrm{m})$, areia fina $(125 \mu \mathrm{m})$, areia muito fina $(65 \mu \mathrm{m})$ e lama $(<65 \mu \mathrm{m})$ de acordo com a escala de FoLK (1974)

As informações sobre a data da desova e a data de eclosão, o sucesso da eclosão, a duração da incubação, a profundidade da câmara de ovos, o número de filhotes vivos, filhotes mortos e ovos inviáveis foram coletadas rotineiramente pela equipe técnica do Projeto TAMARIBAMA que monitorou diariamente a praia. Maiores detalhes sobre estes parâmetros são apresentados por Marcovaldi \& Laurent (1996) e Baptistotte et al. (2003). A duração da incubação é o período transcorrido entre a desova e a saída dos filhotes dos ninhos. O sucesso da 
eclosão é a relação entre o número total de ovos e o número de filhotes vivos (considerando-se apenas aqueles filhotes que conseguem emergir do ninho). A data da desova é o dia em que ocorreu a postura e foi coletada para todos os ninhos.

A comparação entre as diferentes estações reprodutivas foi avaliada por análise de variância (ANOVA). Também foi realizado o estudo comparativo entre os dois ambientes geomorfológicos: praia e berma, considerando os dados de todas as estações reprodutivas. Quando esta análise acusou diferença significativa, procedeu-se ao teste entre as estações reprodutivas. Foi feita, também, uma análise de correlacão entre os parâmetros avaliados em todas as estações reprodutivas. Para avaliar se o número de desovas se distribuiu de maneira semelhante ou não entre a praia e a berma, ao longo das estações reprodutivas, foi aplicado o teste binomial. O tamanho dos sedimentos foi dividido em oito frações granulométricas e cada fração foi considerada como uma variável independente. Correlações detectadas com qualquer uma das frações granulométricas testadas foram consideradas como influência do tamanho do sedimento no parâmetro analisado.

\section{RESULTADOS}

As temporadas reprodutivas tiveram início em outubro e se estenderam até março, com o pico de desovas nos meses de novembro e dezembro, e o auge da eclosão em janeiro e fevereiro. Foram monitorados 132 ninhos durante as quatro temporadas reprodutivas de 2001/2002 a 2004/2005 (Tab. I). O tamanho dos sedimentos, a profundidade do topo da câmara de ovos, o sucesso da eclosão e a duração da incubação variaram entre as estações reprodutivas. Como os parâmetros variaram ao longo dos anos, as temporadas reprodutivas foram analisadas individualmente. Somente a data da desova $(\mathrm{p}=0,848)$, a profundidade da base da câmara de ovos e a altura dos ninhos em relação ao nível do mar não variaram entre as estações reprodutivas. Isso significa que o momento da desova, ou o período do ano em que foram realizadas as posturas, foi o mesmo ao longo das quatro estações reprodutivas avaliadas. Com relação ao tamanho do sedimento, somente as frações areia fina e areia muito fina não mostraram diferença significativa entre as estações reprodutivas analisadas.

Análise das correlações com a distribuição espacial dos ninhos. As desovas se distribuíram de forma diferente entre a praia e a berma apenas na temporada reprodutiva de 2001/2002 ( $p=0,012$ ). Nos outros anos, o teste binomial apontou diferenças não significativas $(\mathrm{p}=0,461 ; \mathrm{p}=0,472$ e $p=0,064,2002 / 2003,2003 / 2004,2004 / 2005$, respectivamente). A maior concentração de ninhos ocorreu nas proximidades da transição da praia com a berma, dentro do limite da praia aberta (Fig. 1).

O tamanho dos sedimentos, a altura dos ninhos em relação ao nível do mar, a profundidade da câmara de ovos e o número de ovos apresentaram variações entre a berma e a praia aberta (Tab. II). A data da desova não variou entre as duas áreas $(\mathrm{p}=0,561)$.

A duração da incubação e o sucesso da eclosão não variaram entre a berma e a praia (Tab. III). A duração da incubação apresentou valores muito próximos nos dois ambientes sugerindo que a razão sexual foi a mesma ao longo das duas áreas de desova.

A data da desova afetou a duração da incubação nas temporadas reprodutivas de 2001/2002 (r=0,687; $\mathrm{p}<0,01), 2002 / 2003$ ( $\mathrm{r}=-0,443 ; \mathrm{p}<0,01)$ e $2004 / 2005$ $(\mathrm{r}=-0,685 ; \mathrm{p}<0,01)$, mas não afetou na temporada de 2003/2004 (r=-0,122; $\mathrm{p}=016$ ).

Análise das correlações com o tamanho do sedimento. O tamanho do sedimento variou entre a praia e a berma quando as temporadas foram consideradas em conjunto (Tab. II) e também quando as temporadas foram analisadas individualmente (Tab. IV). Como conseqüência do retrabalhamento eólico os sedimentos da berma são mais finos e melhor selecionados que os sedimentos da praia aberta.

O tamanho do sedimento exerceu pequena influência sobre os parâmetros reprodutivos quando os dados foram tomados em conjunto ao longo das quatro estações reprodutivas. Evidenciou-se uma alta correlação entre as frações granulométricas, mas essas frações afetaram apenas, e muito pouco, o sucesso da eclosão nas frações areia muito grossa $(r=-0,319 ; \mathrm{p}=0,002)$ e areia

Tabela I. Variação dos parâmetros reprodutivos de Caretta caretta (Linnaeus, 1758) na Praia da Guanabara, Anchieta, Espírito Santo, ao longo de quatro temporadas reprodutivas (média \pm erro padrão; tamanho da amostra). Em cada linha, médias seguidas de letras distintas, na mesma posição, apresentam diferenças significativas no nível de 5\%, segundo a metodologia de Scheffe.

\begin{tabular}{|c|c|c|c|c|c|c|}
\hline Parâmetro & $2001 / 2002$ & $2002 / 2003$ & $2003 / 2004$ & $2004 / 2005$ & Todos os anos & $\mathrm{p}$ \\
\hline Sucesso da eclosão & $70,5^{\mathrm{a}} \pm 4,1(46)$ & $78,2^{\mathrm{ab}} \pm 3,2(36)$ & $72,9^{\mathrm{abd}} \pm 6,6(19)$ & $57,5^{\text {acd }} \pm 6,7(31)$ & $69,9 \pm 2,9(132)$ & 0,031 \\
\hline Duração da incubação & $62,8^{a} \pm 1(37)$ & $58,7^{\mathrm{bc}} \pm 0,7(34)$ & $68,3^{\text {bde }} \pm 0,7$ (17) & $64,3^{\text {ade }} \pm 1,4(22)$ & $62,7 \pm 0,6(110)$ & $<0,01$ \\
\hline Altura do ninho & $292 \pm 8(46)$ & $269 \pm 6,9(39)$ & $284 \pm 16(17)$ & $299 \pm 9,4(27)$ & $285 \pm 4,6(129)$ & 0,088 \\
\hline Profundidade (topo) & $29,6^{\mathrm{a}} \pm 2,1(30)$ & $38,6^{\mathrm{bc}} \pm 1,9(34)$ & $33^{\text {acd }} \pm 2(11)$ & $37,4^{\mathrm{acd}} \pm 2,3(21)$ & $43,9 \pm 1,1(96)$ & 0,006 \\
\hline Profundidade (base) & $53 \pm 1,5(29)$ & $56,1 \pm 1,6(34)$ & $51,9 \pm 3,1(11)$ & $52,5 \pm 25(22)$ & $53,8 \pm 1(96)$ & 0,398 \\
\hline Total de ovos & $136^{\mathrm{a}} \pm 3,2(42)$ & $130^{\mathrm{ac}} \pm 3,5(35)$ & $132^{\text {ace }} \pm 4,9(18)$ & $106^{\text {bdf }} \pm 6,6(25)$ & $128 \pm 2,4(120)$ & $<0,01$ \\
\hline \multicolumn{7}{|l|}{ Tamanho do sedimento } \\
\hline Areia muito grossa & $2,4^{\mathrm{a}} \pm 0,79(23)$ & $4,7^{\mathrm{ac}} \pm 0,83$ & $2,4^{\mathrm{acd}} \pm 0,63(16)$ & $5,9^{\text {bce }} \pm 1,25(26)$ & $4,1 \pm 0,49(97)$ & 0,018 \\
\hline Areia grossa & $41,7^{\mathrm{a}} \pm 3,24(23)$ & $54,8^{\mathrm{bc}} \pm 2,32(32)$ & $39,8^{\text {ade }} \pm 3,93(16)$ & $48,1^{\text {ace }} \pm 3,38(26)$ & $47,4 \pm 1,65(97)$ & 0,005 \\
\hline Areia média & $27,1^{\mathrm{a}} \pm 1,35(23)$ & $18,3^{\mathrm{bc}} \pm 1,75$ & $26^{\mathrm{adc}} \pm 1,75(16)$ & $18,2^{\text {bef }} \pm 1,05(26)$ & $21,6 \pm 0,77(97)$ & $<0,01$ \\
\hline Areia fina & $27,5 \pm 3,4(23)$ & $20,8 \pm 1,86(32)$ & $30,5 \pm 2,87(16)$ & $25,9 \pm 3,62(26)$ & $25,3 \pm 1,51(97)$ & 0,128 \\
\hline Areia muito fina & $1,2 \pm 0,23(23)$ & $1,1 \pm 0,1$ & $1,2 \pm 0,13(16)$ & $1,2 \pm 0,2(26)$ & $1,17 \pm 0,08$ & 0,939 \\
\hline
\end{tabular}






Fig. 1. Distribuição dos ninhos de Caretta caretta (Linnaeus, 1758) na Praia da Guanabara, Anchieta, Espírito Santo, nas temporadas reprodutivas de 2001/2002 a 2004/2005. média $(\mathrm{r}=0,249 ; \mathrm{p}=0,017)$. Nesta análise, foram excluídos os ninhos transferidos. O sucesso da eclosão foi menor nos ninhos situados nos sedimentos mais grossos. A duração da incubação não apresentou correlação com o tamanho dos sedimentos. Ninhos com maior duração de incubação apresentaram um menor sucesso da eclosão $(\mathrm{r}=-0,254 ; \mathrm{p}=0,019)$.

Entretanto, quando os dados foram analisados ano a ano, considerando cada temporada reprodutiva como uma unidade independente, os resultados foram parcialmente diferentes para a duração da incubação. A duração da incubação foi afetada pelo tamanho do sedimento em 2003/2004. Nesta temporada as frações areia grossa $(\mathrm{r}=-0,554 ; \mathrm{p}=0,032)$ e areia fina $(\mathrm{r}=0,615$; $\mathrm{p}=0,015)$ indicam que quanto maior o tamanho do sedimento menor a duração da incubação. O sucesso da eclosão não foi afetado pelo tamanho do sedimento. Nesta análise, foram utilizados apenas os ninhos que completaram a incubação. Foram excluídos do cálculo os ninhos transferidos por causa da erosão da praia. Na temporada de 2004/2005 a duração da incubação se correlacionou negativamente com o sucesso da eclosão $(r=-0,645 ; p=0,002)$ indicando que os ninhos de incubação longa apresentaram um menor número de filhotes vivos.

Análise das correlações com a compartimentação geomorfológica. A porção sul da praia da Guanabara que concentrou $70 \%$ dos ninhos pode ser dividida em dois setores com base nas suas características geológicas (Fig. 1). O setor mais ao sul apresenta um comportamento deposicional favorecido pela proximidade de afloramentos rochosos que minimizam a ação das correntes vindas do sul. Não são verificados indícios de erosão e a praia apresenta uma inclinação suave $\left(3^{\circ}\right) \mathrm{em}$ direção ao mar. A transição da praia aberta para a zona vegetada é suave e marcada pela presença de uma berma que varia de 1 a $3 \mathrm{~m}$ de largura e é um pouco mais alta que a praia adjacente. Neste setor da praia da Guanabara, a praia aberta localiza-se $274 \pm 39 \mathrm{~cm}(\mathrm{n}=29)$ acima do nível do mar e a berma a $305 \pm 39 \mathrm{~cm}(\mathrm{n}=29)$. A berma, apesar de ser limitada em termos de área é muito procurada como local de desova. A berma apresenta sedimentos mais finos e melhor selecionados que a praia aberta (Tab. II) devido ao retrabalhamento eólico. $\mathrm{O}$ transporte pelo vento ainda é responsável por um maior arredondamento e aspecto fosco e cor clara da areia. A vegetação da berma é pouco diversificada e constituída principalmente por Ipomoea pescaprae e I. littorale e em menor proporção Blutaparon portulacoides (Amaranthaceae), Panicum racemosum (Poaceae) e Remirea maritima (Cyperaceae). Durante os quatro anos de monitoramento apenas três ninhos se localizaram na restinga da praia da Guanabara e esses ninhos não foram considerados nas análises.

No setor norte, o comportamento da praia é erosivo e a morfologia é marcada por degraus que constituem pequenos terraços nos meses de janeiro (Fig. 1). No início da temporada reprodutiva a morfologia da praia é em rampa com uma inclinação em torno $7^{\circ}$ e que permite que as fêmeas atinjam a restinga e a berma. Com a proximidade do verão e com as alterações nas correntes litorâneas os sedimentos anteriormente depositados passam a ser retrabalhados gerando as feições erosivas. Neste local pode ocorrer a erosão dos ninhos localizados na praia aberta. Os degraus gerados pela erosão chegam a mais de $2 \mathrm{~m}$ de altura. Os ninhos da praia aberta situaram-se $251 \pm 43 \mathrm{~cm}(\mathrm{n}=17)$ acima do nível do mar e os da berma a 
Tabela II. Variação entre a praia aberta e a berma dos parâmetros reprodutivos e fisiográficos das áreas utilizadas para a desova de Caretta caretta (Linnaeus, 1758) na Praia da Guanabara, Anchieta, Espírito Santo, ao longo de quatro temporadas reprodutivas (média \pm erro padrão; tamanho da amostra).

\begin{tabular}{lccc}
\hline Parâmetro & praia & berma & $p$ \\
\hline Sucesso da eclosão & $65,4 \pm 4,3(69)$ & $75,1 \pm 2,5(61)$ & 0,058 \\
Duração da incubação & $62,2 \pm 0,9(51)$ & $63,1 \pm 0,8(57)$ & 0,472 \\
Altura do ninho & $265 \pm 5,6(66)$ & $307 \pm 6,4(63)$ & $<0,01$ \\
Profundidade (topo) & $40,1 \pm 1,4(49)$ & $29,2 \pm 1,5(45)$ & $<0,01$ \\
Profundidade (base) & $58 \pm 1,5(49)$ & $49,8 \pm 0,7(45)$ & $<0,01$ \\
Total de ovos & $124 \pm 3,6(57)$ & $133 \pm 2,8(61)$ & 0,04 \\
Tamanho do sedimento & & $2,1 \pm 0,3(51)$ & $<0,01$ \\
Areia muito grossa & $6,2 \pm 0,89(46)$ & $40,7 \pm 2,3(51)$ & $<0,01$ \\
Areia grossa & $54,9 \pm 1,86(46)$ & $25,3 \pm 0,97(51)$ & $<0,01$ \\
Areia média & $17,5 \pm 0,9(46)$ & $304 \pm 2(51)$ & $<0,01$ \\
Areia fina & $19,7 \pm 1,96$ & $1,3 \pm 0,1(51)$ & 0,065 \\
Areia muito fina & $1 \pm 0,1(46)$ & &
\end{tabular}

Tabela III. Variação da duração da incubação e sucesso da eclosão de Caretta caretta (Linnaeus, 1758) na Praia da Guanabara, Anchieta, Espírito Santo, ao longo de quatro temporadas reprodutivas (média \pm desvio padrão; tamanho da amostra).

\begin{tabular}{|c|c|c|c|c|c|c|}
\hline \multirow[t]{2}{*}{ Temporada } & \multicolumn{3}{|c|}{ Duração da incubação } & \multicolumn{3}{|c|}{ Sucesso da eclosão } \\
\hline & praia & berma & $\mathrm{p}$ & praia & berma & $\mathrm{p}$ \\
\hline $2001 / 2002$ & $64 \pm 5,98$ (14) & $62,1 \pm 5,93$ & 0,3495 & $67,1 \pm 35,8$ & $73,2 \pm 19,4(26)$ & 0,4653 \\
\hline $2002 / 2003$ & $58,3 \pm 5,21$ & $59,4 \pm 2,27$ & 0,4825 & $79,2 \pm 22,1$ & $76,8 \pm 15,4(15)$ & 0,7191 \\
\hline $2003 / 2004$ & $69,5 \pm 2,38$ & $67,9 \pm 3,17$ & 0,3774 & $54,53 \pm 42,4(6)$ & $81,2 \pm 16,6$ & 0,0644 \\
\hline $2004 / 2005$ & $64,1 \pm 5,19$ & $64,7 \pm 9,78$ & 0,8494 & $53,4 \pm 40,1$ & $67,8 \pm 29,6(7)$ & 0,4012 \\
\hline Todos os anos & $62,2 \pm 6,21(51)$ & $63,1 \pm 6,08(57)$ & 0,4724 & $65,4 \pm 35,6(69)$ & $75,2 \pm 19,3(61)$ & 0,0582 \\
\hline
\end{tabular}

Tabela IV. Características dos sedimentos da fração arenosa da praia e da berma na Praia da Guanabara, Anchieta, Espírito Santo, ao longo das estações reprodutivas (média \pm erro padrão).

\begin{tabular}{|c|c|c|c|c|c|c|c|c|c|c|c|c|}
\hline \multirow[t]{2}{*}{ Fração } & \multicolumn{3}{|c|}{2001} & \multicolumn{3}{|c|}{2002} & \multicolumn{3}{|c|}{2003} & \multicolumn{3}{|c|}{2005} \\
\hline & praia & berma (15) & $\mathrm{p}$ & ) & berma & $\mathrm{p}$ & praia (4) & berma (12) & $\mathrm{p}$ & praia (18) & berma (8) & $\mathrm{p}$ \\
\hline Areia $m$ & $3,9 \pm 1,94$ & $1,5 \pm 0,25$ & 0,065 & $7 \pm 1,45$ & $2,7 \pm 0,47$ & 0,019 & $5,4 \pm 1,55$ & $1,4 \pm 0,35$ & 0,003 & $7,1 \pm 1,64$ & $3,4 \pm 1$ & 138 \\
\hline Areia $g$ & $56,1 \pm 2,09$ & $34 \pm 3,46$ & 0,001 & $59,9 \pm 1,85$ & $49,7 \pm 3,91$ & 0,036 & $56,5 \pm 1,17$ & $34,3 \pm 4,09$ & 0,015 & $49,7 \pm 4,11$ & $44,8 \pm 6,17$ & 0,466 \\
\hline Areia $n$ & $24 \pm 2,1$ & $28,8 \pm 1,62$ & 0,098 & $15,9 \pm 1,5$ & $21,6 \pm 1,71$ & 0,007 & $18,4 \pm 2,18$ & $28,6 \pm 1,66$ & 0,005 & $16,7 \pm 1,27$ & $21,5 \pm 1,36$ & 0,04 \\
\hline Arei & $15 \pm$ & $34 \pm 4,02$ & 0,002 & $16,8 \pm 1,74$ & $24,8 \pm 3,02$ & 0,102 & 18, & 34, & 08 & 39 & 28 &, 549 \\
\hline Areia muito fina & $0,6 \pm 0,19$ & $1,5 \pm 0,3$ & 0,007 & $1,2 \pm 0,13$ & $1,1 \pm 0,16$ & 0,551 & $0,8 \pm 0,15$ & $1,32 \pm 0,15$ & 0,064 & $1,17 \pm 0,26$ & $1,18 \pm 0,28$ & 0,758 \\
\hline
\end{tabular}

$354 \pm 41 \mathrm{~cm}(\mathrm{n}=12)$. Os ninhos da praia e da berma localizaram-se em alturas diferentes nas zonas de erosão $(\mathrm{p}<0,001)$ e na zona de acresção $(\mathrm{p}=0,0037)$. Entretanto não existiu diferença significativa na altura dos ninhos $(\mathrm{p}=0,7123)$ quando eles foram tomados em conjunto na zona de erosão (294 \pm 66 cm; n=17) e na zona de deposição $(290 \pm 42 \mathrm{~cm} ; \mathrm{n}=58)$.

\section{DISCUSSÃO}

Os ninhos localizados na berma são mais rasos que os ninhos localizados na praia e essa diferença poderia ser explicada pelas características dos sedimentos e da cobertura vegetal. Os sedimentos da berma, por serem retrabalhados pelo vento, apresentam uma maior seleção granulométrica ao passo que os sedimentos da praia aberta são continuamente remobilizados pela ação das ondas (Suguio, 2003; SouZa et al., 2005). As variações na intensidade das ondas e das correntes litorâneas atuantes na praia aberta não permitem uma seleção granulométrica tão grande quanto na berma. Como resultado, os sedimentos da berma são mais coesos que os da praia aberta. Além das características dos sedimentos, as raízes conferem uma maior coesão aos sedimentos. A maior coesão e compactação da berma facilitam a abertura dos ninhos e fazem com que os ninhos localizados na berma sejam mais rasos que os ninhos da praia. Sedimentos incoesos tendem a colapsar durante a abertura dos ninhos (Bustard \& GreenHam, 1968; Mortimer, 1995). A umidade aumenta com a profundidade aumentando, também, a coesão dos sedimentos, o que permite que a câmara de ovos seja aberta na praia. A umidade adequada à manutenção da parede da câmara de ovos somente estará disponível a partir dos $40 \mathrm{~cm}$ de profundidade e marca o topo dos ninhos na praia aberta (FERREIRA JúNIOR et al., 2003). Na praia da Guanabara, os sedimentos mal selecionados são mais incoesos, ao contrário do que aponta KARAVAS et al. (2005) para a praia de Sekania na Grécia.

Uma vez que as tartarugas utilizaram mais a berma que a praia para a desova, deve-se considerar as diferenças nas condições de acesso e também da área disponível. A praia aberta possui uma área muito maior que a berma que, na praia de Guanabara, apresenta, no 
máximo, $3 \mathrm{~m}$ de largura. $\mathrm{O}$ acesso à berma nem sempre é possível, principalmente nos meses de janeiro e fevereiro, quando o limite praia/berma está escarpado nas partes da praia que apresentam comportamento erosivo. Desta forma, acredita-se que a cobertura vegetal, a compartimentação geomorfológica e as características do sedimento (i.e. tamanho, compactação e seleção granulométrica) são fatores que influenciariam a escolha das áreas de desova de $C$. caretta na praia da Guanabara.

A relação entre o tamanho dos sedimentos e a reprodução de $C$. caretta deve ser feita com cautela, sendo necessárias algumas ponderações. Considerando-se todos os ninhos das quatro temporadas reprodutivas em conjunto, o sucesso da eclosão da berma $(75,1 \pm 2,5$; $n=61)$ é maior que o da praia $(65,4 \pm 4,3 ; n=69)$. Mas quando excluímos os ninhos que seriam erodidos pela ação das marés e foram transferidos (e cujo sucesso de eclosão in situ seria zero), o sucesso da eclosão na praia $(80,5 \pm 2,5 ; n=54)$ passa a ser maior que na berma. Os motivos desta diferença podem se relacionar à menor profundidade dos ninhos que provocaria uma variação de temperatura muito grande nos ovos mais superficiais (Mrosovsky et al., 1992; SouZA \& VoGT, 1994; GodFrey et al., 1996). É relativamente comum, para os ninhos localizados na face de berma, que as duas camadas mais superficiais de ovos não eclodam, em função das variações extremas da temperatura e umidade (MCGEHEE, 1990). Raízes de plantas que se desenvolvem dentro dos ninhos também reduzem o sucesso da eclosão (GoDLEY et al., 2001). Este fato mostra a importância de se analisar todas as variáveis e a dificuldade de se mensurar todos os fatores envolvidos

A alta concentração de ninhos na transição da praia para a berma, a pequena espessura da berma e as grandes diferenças sedimentológicas dessas áreas apontam para as limitações que as técnicas de mapeamento em maior escala oferecem. Mesmo o emprego de GPS na marcação dos ninhos pode levar a resultados insatisfatórios na análise das correlações que afetam a reprodução das tartarugas marinhas. Para uma análise mais detalhada devem-se coletar dados referentes ao comportamento sedimentológico e à compartimentação geomorfológica das praias. O mapeamento de grandes áreas auxilia na determinação de bolsões de desova, mas um maior detalhamento do posicionamento dos ninhos é necessário para analisar a influência dos fatores físicos na reprodução. A utilização de transector (BJORDNAL \& BOLten, 1992; KARAVAs et al., 2005) para a análise do tamanho dos grãos também pode levar a interpretações equivocadas caso não sejam consideradas pequenas variações morfológicas das áreas de sedimentação. A coleta de amostra em todos os ninhos seria desejável, mas pode se tornar inviável em praias com um número muito grande de desovas e onde ocorra a sobreposição de ninhos (GodLey et al., 2001)

Apesar do sucesso da eclosão não ter apresentado variações significativas entre a praia e a berma, projetos de manejo que envolvem a transferência de ninhos devem levar a compartimentação geomorfológica em consideração. A base dessa assertiva considera a multiplicidade de fatores que controlam a incubação (NARO-MACIEL et al., 1999; GARMESTANi et al., 2000; GoDLEy et al., 2001), mas que se reflete, principalmente, na profundidade da câmara de ovos. Existe uma diferença de cerca de $10 \mathrm{~cm}$ na profundidade dos ninhos da berma e da praia aberta. Essa diferença, a menor na berma, compensaria as possíveis diferenças que a cobertura vegetal, o tamanho dos sedimentos (FERREIRA JúnIOR \& CAstro, 2003), a umidade, a cor, o albedo (Hays et al., 2001) e as trocas gasosas (ACKERMAN et al., 1985) podem exercer no ambiente hídrico e termal dos ninhos, levando a resultados semelhantes nos dois compartimentos geomorfológicos. Neste caso, recomenda-se que a transferência dos ninhos, quando inevitável, seja feita para locais com características geológicas similares e não apenas para áreas próximas ou adjacentes. Quando a transferência envolver duas áreas diferentes em termos de cobertura vegetal, tamanho dos sedimentos e compartimentação geomorfológicas, recomenda-se que a profundidade do ninho seja aquela encontrada nos ninhos naturais da área de destino e não a profundidade original do ninho que está sendo transferido.

A data da desova de $C$. caretta está diretamente relacionada à duração da incubação que diminui com a proximidade do verão (MrosovsKY \& ProvanchA, 1989; Marcovaldi et al., 1997; NARO-Maciel et al., 1999; BAPTISTOTTE et al., 1999; FERREIRA JÚNIOR et al., 2003). Mesmo a temporada reprodutiva de 2003/2004 não apresentou essa correlação, corroborando a necessidade de estudos de longa duração (CONGDON et al., 2000). Além do clima, as características do local da desova também afetaram a duração da incubação que variou ao longo dos anos monitorados e correlacionou-se negativamente com o tamanho médio dos sedimentos e com as frações areia grossa e areia muito grossa. Quanto maior o sedimento, menor a duração da incubação. Esse fato pode estar associado ao aumento do tamanho dos sedimentos que diminui a porosidade e aumenta a permeabilidade da areia. A influência que o tamanho do sedimento exerce na duração da incubação é pequena mas significativa e indica que as temporadas reprodutivas devem ser tratadas separadamente. Quando as quatro temporadas foram analisadas em conjunto não se verificou esta correlação, mas quando as temporadas foram analisadas ano a ano, a duração da incubação se mostrou influenciada pelo tamanho do sedimento em 2003/2004. A maneira como as estações reprodutivas são analisadas (se em conjunto ou individualmente) influencia os resultados.

A duração da incubação não variou entre a praia e a berma apesar das diferenças significativas dos sedimentos. A diferença na cobertura vegetal e na profundidade dos ninhos pode explicar este fato. Para tartarugas de água doce (Podocnemis expansa (Schweigger, 1812)), ninhos situados em sedimentos mais finos apresentam até 14 dias de diferença para ninhos localizados em praia de sedimentos grossos (FERREIRA JúNIOR \& CASTRO, 2003). Os ninhos da berma, apesar de possuírem sedimentos mais finos não diferem na duração da incubação da praia porque são mais rasos e cobertos por uma vegetação rasteira. A cobertura vegetal e a menor profundidade dos ninhos minimizam a menor transferência de calor em profundidade, fazendo com que a temperatura da câmara de ovos nos dois compartimentos geomorfológicos seja semelhante (Mrosovsky et al., 1992; GodFrey et al., 1996; NAROMACIEL et al., 1999). Este fato deve ser observado nos programas que envolvem a transferência de ninhos. Os ninhos transferidos para berçários localizados na face de berma (onde os sedimentos são mais finos e bem selecionados) devem ter uma profundidade menor que 
aqueles berçários localizados na face de praia. Estas conclusões vêm se somar àquelas de NARO-MACIEL et al. (1999) que apontaram para o fato de que uma escolha criteriosa de berçários artificiais para onde os ninhos são transferidos não afeta a razão sexual dos filhotes de $C$. caretta.

Agradecimentos. Ao Projeto TAMAR-IBAMA, à Prefeitura Muncipal de Anchieta e à Samarco Mineração pelo apoio na coleta dos dados e infra-estrutura da Base de Anchieta. Agradecimentos especiais aos monitores e estagiários e equipe técnica que auxiliaram no monitoramento dos ninhos e na coleta dos sedimentos.

\section{REFERENCIAS BIBLIOGRÁFICAS}

Ackerman, R. A.; Seagrave, R. C.; Dmi'el, R. \& Ar, A. 1985. Water and heat exchange between parchment-shelled reptile eggs and their surroundings. Copeia 1985:703-711.

Baptistotte, C.; Scalfoni, J. T. \& Mrosovsky, N. 1999. Maleproducing thermal ecology of a southern loggerhead nesting beach in Brazil: implications for conservation. Animal Conservation 2(1):9-13.

Baptistotte, C.; Thomé, J. C. A. \& Buordnal, K. A. 2003. Reproductive biology and conservation status of the loggerhead sea turtle (Caretta caretta) in Espírito Santo State, Brazil. Chelonian Conservation and Biology 4(3):523-529.

Bjorndal, K. A. \& Bolten, A. B. 1992. Spatial distribution of green turtle (Chelonia mydas) nests at Tortuguero, Costa Rica. Copeia 1992(1):45-53.

Bobyn, M. L. \& BRooks. R. J. 1994. Incubation conditions as potential factors limiting the northern distribution of snapping turtles, Chelydra serpentina. Canadian Journal of Zoology 72(1):28-37.

Bustard, H. R. \& Greenham, P. 1968. Physical and chemical factors affecting hatching in the green sea turtle, Chelonia mydas (L.). Ecology 49(2):269-276.

Congdon, J. D.; Nagle, R. D.; Kinney, O. M.; Osentoski, M.; Avery, H. W.; van Loben, C. R. S. \& Tinckle, D. W. 2000. Nesting ecology and embryo mortality: implications for hatchling success and demography of Blanding's turtles (Emydoidea blandingii). Chelonian Conservation and Biology 3:569-579.

Ferreira Júnior, P. D. \& Castro, P. T. A. 2003. Geological control of Podocnemis expansa and Podocnemis unifilis nesting areas in Javaés River, Bananal Island, Brazil. Acta Amazonica 33(3):445-468.

Ferreira Júnior, P. D.; Castro, P. T. A.; Addad, J. E. \& Lorenzo, M. 2003. Aspectos fisiográficos das áreas de nidificação da tartaruga marinha Caretta caretta na praia da Guanabara, Anchieta, Espírito Santo. Publicações Avulsas do Instituto Pau Brasil de História Natural 7:25-40.

Foley, M. A.; Peck, A. S.; Harman, R. G. \& Richardson, L.W. 2000. Loggerhead turtle (Caretta caretta) nesting habitat on low-relief mangrove island in southwest Florida and consequences to hatchling sex ratios. Herpetologica 56(4): 433-445.

Folk, R. L. 1974. Petrology of sedimentary rocks. Austin, Hemphill. 182p.

Fowler, L. E. 1979. Hatching success and nest predation in the green sea turtle, Chelonia mydas, at Tortuguero, Costa Rica. Ecology 60(5):946-955.

Garmestani, A. S.; Percival, H. F.; Portier, K. M. \& Rice, K. G. 2000. Nest-site selection by loggerhead sea turtle in Florida's Ten Thousand Island. Journal of Herpetology 34(4):504-510.

Godfrey, M. H.; BarReto, R. \& Mrosovsky, N. 1996. Estimating past and present sex ratios of sea turtles in Suriname. Canadian Journal of Zoology 74:267-277.

Godley, B. J.; Broderick, A. C. \& Mrosovsky, N. 2001. Estimating hatchling sex ratios of loggerhead turtles in Cyprus from incubation durations. Marine Ecology Progress Series 210: $195-201$

Hays, G. C. \& Speakman, J. R. 1993. Nest placement by loggerhead turtles, Caretta caretta. Animal Behaviour 45(1):47-53.
Hays, G. C.; Ashworth, J. S.; Barnsley, M. J.; Broderick, A. C.; Emery, D. R.; Godley, B. J.; Henwood, A. \& Jones, E. L. 2001. The importance of sand albedo for the thermal conditions on sea turtle nesting beaches. Oikos 93(1):87-94.

Horrocks, J. A. \& SсотT N. M. 1991. Nest site location and nest success in the hawksbill turtle Eretmochelys imbricata in Barbados, West Indies. Marine Ecology Progress Series 69(1):1-8.

Karavas, N.; Georghiou, K.; Arianoutsou, M. \& Dimopoulos, D. 2005. Vegetation and sand characteristics influencing nesting activity of Caretta caretta on Sekania beach. Biological Conservation 121:177-188.

Marcovaldi, M. A. \& Laurent, A. 1996. A six-season study of marine turtle nesting at Praia do Forte, Bahia, Brazil, with implications for conservation and management. Chelonian Conservation and Biology 2(1):55-59.

Marcovaldi, M. Â.; Godfrey, M. H. \& Mrosovsky, N. 1997. Estimating sex ratios of loggerhead turtles in Brazil from pivotal incubation durations. Canadian Journal of Zoology 75:755-770.

McGenee, M. A. 1990. Effects of moisture on eggs and hatchlings of loggerhead sea turtle (Caretta caretta). Herpetologica 46:251-258

Miller, J. D. 1997. Reproduction in sea turtles. 1997. In: LuTZ, P.; Musik, J. \& Wynekan, J. eds. Biology of sea turtles, v.2, CRC, p.51-81.

Milton, S. L.; Schulman, A. A. \& Lutz, P. L. 1997. The effect of beach nourishment with aragonite versus silicate sand on beach temperature and loggerhead sea turtle nesting success. Journal of Coastal Research 13:904-915.

Mortimer, J. A. 1990. The influence of beach sand characteristics on the nesting behavior and clutch survival of green turtles (Chelonia mydas). Copeia 1990(3):802-817.

. 1995. Factors influencing beach selection by nesting sea turtles. In: BJoRdnal, K. A. ed. Biology and conservation of sea turtles. Washington, Smithsonian Institution. p.45-51.

Mrosovsky, N. \& YnTema, C. L. 1980. Temperature dependence of sexual differentiation in sea turtles: implications for conservation practices. Biological Conservation 18:271-280.

Mrosovsky, N.; Dutton, P. H. \& Whitmore, C. P. 1984. Sex ratio of two species of sea turtle nesting in Suriname. Canadian Journal of Zoology 62:2227-2239.

Mrosovsky, N. \& Provancha, J. 1989. Sex ratio of loggerhead sea turtles hatching on a Florida beach. Canadian Journal of Zoology 67:2533-2539.

Mrosovsky, N.; Bass, A.; Corliss, L. A.; Richardson, J. I. \& RichaRdSON, T. H. 1992. Pivotal and beach temperatures for hawksbill turtles nesting in Antigua. Canadian Journal of Zoology 70(10):1920-1925.

Mrosovsky, N.; Baptistotte, C. \& Godfrey, M. H. 1999. Validation of incubation duration as an index of the sex ratio of hatchling sea turtles. Canadian Journal of Zoology 77:831-835.

Naro-Maciel, E.; Mrosovsky, N. \& Marcovaldi, M. Â. 1999. Thermal profiles of sea turtle hatcheries and nesting areas at Praia do Forte, Brazil. Chelonian Conservation and Biology 3:407-413.

SouzA, R. R. \& Vogt, R. C. 1994. Incubation temperature influences sex and hatchling size in the neotropical turtle Podocnemis unifilis. Journal of Herpetology 28:453-464.

Souza, C. R. G.; Suguio, K.; Oliveira, A. M. S. \& Oliveira, P. E. 2005. Quaternário do Brasil. Ribeirão Preto, Holos. 382p

Spotila, J. R.; Standora, E. A.; Morreale, S. J. \& Ruiz, G. 1987. Temperature dependent sex determination in the green turtle (Chelonia mydas): effects on the sex ratio on a natural nesting beach. Herpetologica 43:74-81.

Suguio, K. 2003. Geologia sedimentar. São Paulo, Edgard Blücher. 400p

Wibbels, T. 2003. Critical approaches to sex determination in sea turtle biology and conservation. In: Lutz, P.; MusiK, J. \& WYNEKAN, J. eds. Biology of sea turtles, v.2, CRC, p.103-134. Wood, D. W. \& BJordnal, K. A. 2000. Relation of temperature, moisture, salinity, and slope to nest site selection in loggerhead sea turtles. Copeia 2000(1):119-128. 